\title{
Review
}

\section{Dynamics of mitochondrial morphology in healthy cells and during apoptosis}

\author{
M Karbowski ${ }^{1}$ and RJ Youle ${ }^{\star, 1}$ \\ 1 The Biochemistry Section, SNB, NINDS, NIH, Bethesda, MD, USA \\ * Corresponding author: RJ Youle; The Biochemistry Section, SNB, NINDS, \\ $\mathrm{NIH}$, Building 10, Room 5D-37, 10 Center Drive, Bethesda, MD 20892, USA; \\ E-mail: youle@ helix.nih.gov
}

Received 6.2.03; revised 10.3.03; accepted 19.3.03

Edited by G Melino

\begin{abstract}
Mitochondria exist as dynamic networks that often change shape and subcellular distribution. The number and morphology of mitochondria within a cell are controlled by precisely regulated rates of organelle fusion and fission. Recent reports have described dramatic alterations in mitochondrial morphology during the early stages of apoptotic cell death, a fragmentation of the network and the remodeling of the cristae. Surprisingly, proteins discovered to control mitochondrial morphology appear to also participate in apoptosis and proteins associated with the regulation of apoptosis have been shown to affect mitochondrial ultrastructure. In this review the recent progress in understanding the mechanisms governing mitochondrial morphology and the latest advances connecting the regulation of mitochondrial morphology with programmed cell death are discussed.

Cell Death and Differentiation (2003) 10, 870-880. doi:10.1038/ sj.cdd. 4401260

Keywords: Bax; fission; fusion; fragmentation; Drp1; Fzo1
\end{abstract}

\section{Introduction}

\section{Dynamics of mitochondria in living cells: fission and fusion maintain homeostasis}

Mitochondria are dynamic organelles that can change in number and morphology within a cell during development, the cell cycle or when challenged with various toxic conditions. Imaging of living cells stained with vital, cationic fluorescent probes, such as Rhodamine 123 and JC-1, or green fluorescent proteins specifically targeted to mitochondria revealed that mitochondria are highly dynamic, motile and undergo frequent shape changes (Figure 1). ${ }^{1-3}$ The classical 'textbook' view, based mainly on two-dimensional electron micrographs, of mitochondria as bean-shaped structures with a highly folded inner membrane (IMM) forming mitochondrial cristae enveloped by a relatively uncomplicated outer membrane (OMM) has been challenged since the introduction of $3 D$ reconstitution technologies. The presence of dynamic, tubular, $28 \mathrm{~nm}$ junctions connecting cristae to the IMM was found by serial sectioning and EM tomography. ${ }^{4}$ In addition, a network of interconnected, tubular mitochondria has been detected by high-resolution $3 \mathrm{D}$ imaging of mitochondria expressing green fluorescent protein targeted to the mitochondrial matrix (mito-GFP). ${ }^{5}$

Several other lines of evidence suggest that, at least under some conditions, mitochondria in mammalian cells can form physically interconnected networks ${ }^{6,7}$ that may represent an efficient system to deliver energy, or channel calcium between different areas of the cell. ${ }^{6,7}$ High connectivity of these organelles within the cell has been supported by the fact that, in cells stained with mitochondria specific, membrane potential $\left(\Delta \Psi_{\mathrm{m}}\right)$-sensitive fluorescent probes, depolarizing stimuli targeted to a small area of the mitochondrion, leads to the collapse of the $\Delta \Psi_{\mathrm{m}}$ within the network over the length of tens of micrometers. ${ }^{6,7}$ Moreover, when fluorescence recovery after photobleaching (FRAP) has been applied, photobleaching of mito-GFP within a small area of the cell resulted in the sequential redistribution of the mito-GFP from unaffected portions of the cell, ${ }^{5}$ suggesting lumenal continuity of the mitochondria within network(s) over broad areas of the cell.

Frequent fusion and fission within a dynamic network may be an efficient means of intermitochondrial DNA (mtDNA) complementation through exchange of genomes between fusing mitochondria. ${ }^{8}$ Introduction of mitochondria bearing mtDNA mutations that inactivate cytochrome coxidase (COX) into cells with wild-type mitochondria, resulted in dynamic mixing of matrix and membrane proteins, as well as mtDNA transfer, between normal- and COX-activity-deficient organelles so that all mitochondria within the cell displayed comparable, high COX activity. mtDNA is prone to mutations 
$0^{\prime}$

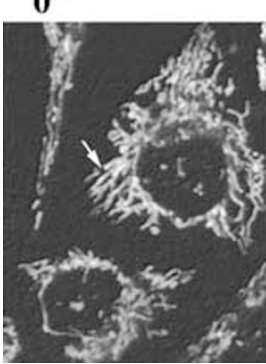

$\mathbf{5}^{\prime}$

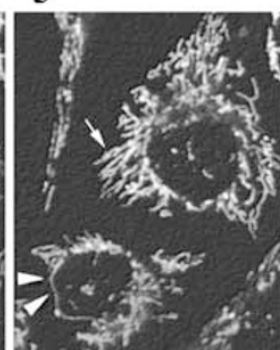

$10^{\prime}$

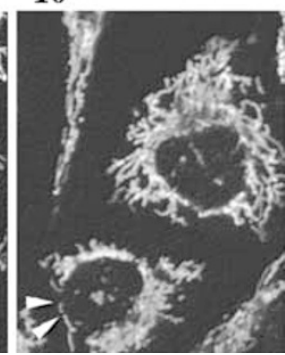

Figure 1 Dynamic network-like behavior of mitochondria in cultured mammalian cells. HeLa cells were stained with the mitochondria-specific fluorescent probe Mitotracker Red CMXRos and imaged over time (at time 0 , followed by 5 and $10 \mathrm{~min}$ ) using confocal microscopy. In addition to high mobility and frequent shape changes, fusion (arrows) and fission (arrowheads) of mitochondria can be detected

that accumulate in aging individuals. Genetic complementation, likely achievable due to the dynamic network-like behavior, may represent a mechanism of repairing mtDNA mutations, and consequently maintain properly functioning oxidative complexes within the cell. In yeast, serial sectioning and reconstruction of electron micrographs as well as experiments with organisms expressing mito-GFP revealed also the presence of a single branching mitochondrion. ${ }^{9-11}$

In addition to their appearance as network-like, functionally united organelles, mitochondria also can be morphologically and functionally independent within cells. ${ }^{12,13}$ It has been demonstrated that in several cell lines, including HeLa cells used for the FRAP experiments described above, mitochondria exist as a heterogeneous mixture of elongated, tubular and short, vesicular forms, which may differ within one cell in the $\Delta \Psi_{\mathrm{m}}$, the rate of permeability transition pore (PTP) activation and the dynamics of $\mathrm{Ca}^{2+}$ sequestration. ${ }^{12,13}$ In living Saccharomyces cerevisiae expressing mito-GFP and imaged using high-resolution-multifocal multiphoton-confocal microscopy, the number of branch points of the mitochondria was found to be regulated by the growth conditions and increased about four times when the yeast were grown on a nonfermentable carbon source, compared to growth on glucose. ${ }^{9}$ Therefore, the discrepancies in the morphology of the mitochondria described by different laboratories using the same cell lines may result from different culture conditions.

A decrease in connectivity and formation of short, round mitochondria occurs under conditions that compromise mitochondrial function, such as mtDNA depletion, ${ }^{14}$ or treatment with mitochondrial toxins. ${ }^{15}$ These shifts from highly branched to fragmented morphologies of mitochondria are most likely regulated by the rates of fission and fusion events. Time-lapse experiments reveal that the frequency of fusion and fission events in yeast mitochondria is equal ${ }^{11}$ and serves as an efficient means of maintaining mitochondrial number and morphology. Consequently, inhibition of both processes does not affect mitochondrial morphology whereas genetic inactivation of the fusion step leads to fragmentation and genetic inactivation of fission results in an increase in mitochondrial connectivity. ${ }^{16,17}$ Under different growth conditions, fission or fusion rates may change and lead to an increase or decrease in mitochondrial number. Until recently,
Proteins regulating mitochondrial fission

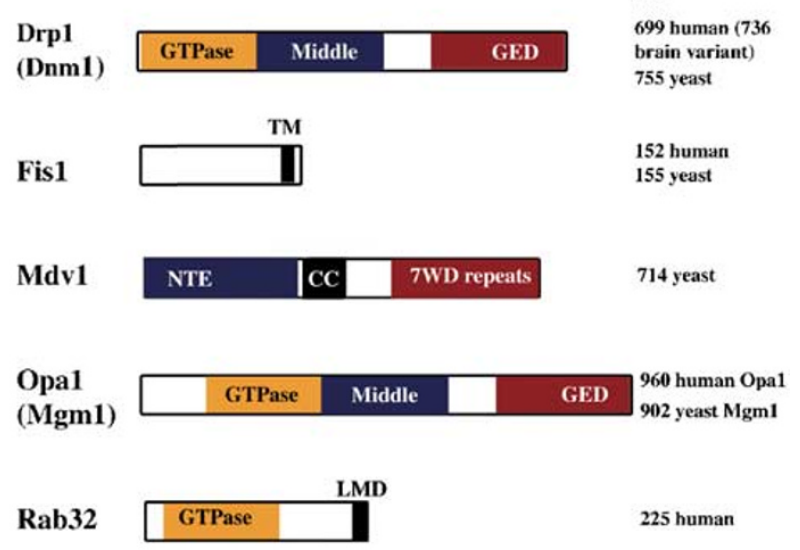

Proteins regulating mitochondrial fusion

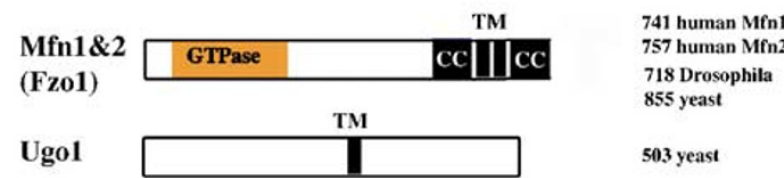

Figure 2 Primary structures of proteins participating in the regulation of mitochondrial morphology. Abbreviations: GTP hydrolysis domain (GTPase), dynamin-homology middle domain (Middle), GTPase effector assembly/domain (GED), transmembrane domain (TM), N-terminal extension (NTE), coiled-coil domain (CC), lipid modifying/membrane attachment domain (LMD). For details see text

little was known about the molecular mechanisms regulating mitochondrial number and morphology. However, combined genetic, biochemical and microscopic approaches have revealed the existence of a number of proteins participating in mitochondrial fusion and fission (Figure 2, Table 1). Discoveries of the components of the fission/fusion machinery in yeast and Drosophila melanogaster now enable us to have the first look at the molecular events governing regulation of the mitochondrial morphology in mammalian cells.

\section{Control of the mitochondrial morphology: GTP-driven machines}

\section{Regulation of the mitochondrial fission}

During proliferation of the budding yeast, S. cerevisiae, efficient transport of mitochondria and other organelles to the daughter bud seems to be essential for the viability of the newly formed cells. Screening of $S$. cerevisiae for mutations resulting in the inhibition of transport to daughter buds and aberrant distribution and morphology of mitochondria led to the discovery of a number of proteins indispensable for proper mitochondrial distribution and maintenance, functionally classified as mitochondrial distribution and maintenance (Mdm) factors. ${ }^{18,19}$ Multiple examples of Mdm mutants are discussed in detail by Yaffe $^{18}$ and only some examples will be discussed here. Inhibition of Mdm10, Mdm12 or the mito- 
Table 1 Proteins participating in the regulation of mitochondrial morphology

\begin{tabular}{|c|c|c|c|c|}
\hline Protein & $\begin{array}{l}\text { Mediated process } \\
\text { (submitochondrial } \\
\text { localization) }\end{array}$ & Organism & Protein characteristics & References \\
\hline Drp1/Dnm1 ${ }^{a}$ & Fission (OMM) & $\begin{array}{l}\text { Human, } \\
\text { C. elegans yeast }\end{array}$ & $\begin{array}{l}\text { GTPase from the dynamin family, localizes to } \\
\text { punctate foci on the OMM; DN mutant blocks } \\
\text { mitochondrial fragmentation and delays apoptosis }\end{array}$ & $\begin{array}{l}16,17,32 \\
33,36-39 \\
58,77,82\end{array}$ \\
\hline Mdv1/Fis2/Gag3/Net2 & Fission (OMM) & Yeast & $\begin{array}{l}\text { WD-repeat protein, coparticipates with Dnm1 in } \\
\text { punctate fission foci }\end{array}$ & $40-43$ \\
\hline Fis1/Mdv2 & Fission (OMM) & Yeast, fly, human & $\begin{array}{l}\text { OMM integral protein, recruits Dnm1 to fission foci; } \\
\text { may also actively participate in membrane scission }\end{array}$ & 42,43 \\
\hline Rab32 & Fission (OMM) & Human & $\begin{array}{l}\text { Small GTPase, member of the Rab family, } \\
\text { colocalizes with mitochondria; serves also } \\
\text { as a kinase A anchoring protein }\end{array}$ & 50 \\
\hline $\operatorname{Mgm} 1 / \mathrm{OPA}^{\mathrm{a}}$ & Fusion (OMM, IMM) ${ }^{\mathrm{b}}$ & $\begin{array}{l}\text { yeast, human, } \\
\text { mouse }\end{array}$ & $\begin{array}{l}\text { GTPase from the dynamin family; potential role in the } \\
\text { regulation of the cristae morphology; downregulation } \\
\text { induces mitochondrial fragmentation, remodeling of } \\
\text { cristae and apoptosis; in humans mutations are } \\
\text { associated with dominant optic atrophy }\end{array}$ & $\begin{array}{l}29-31,34 \\
35,74\end{array}$ \\
\hline Fzo1p/Mfn1\&2 & Fusion (OMM) & $\begin{array}{l}\text { Yeast, fly, human, } \\
\text { mouse }\end{array}$ & $\begin{array}{l}\text { Large GTPase containing C-terminal coiled-coil } \\
\text { domain }\end{array}$ & $51-54$ \\
\hline Ugo1 & Fusion (OMM) & Yeast & Integral protein of the OMM & 59 \\
\hline
\end{tabular}

${ }^{a}$ Proteins with a reported role in the apoptosis. ${ }^{b}$ Submitochondrial localization is controversial.

Table 2 Pro- and antiapoptotic proteins that appear to play a role in morphological remodeling of mitochondria

\begin{tabular}{|c|c|c|c|}
\hline Protein & $\begin{array}{l}\text { Mediated process } \\
\text { (submitochondrial } \\
\text { localization) }\end{array}$ & Protein characteristics & References \\
\hline Bax/Bak & Proapoptotic (OMM) & $\begin{array}{l}\text { Overexpression induces fragmentation of mitochondria; upon induction } \\
\text { of apoptosis they coalesce into submitochondria punctate foci at the } \\
\text { scission sites that colocalize with Drp1 and Mfn2 }\end{array}$ & $75,77,83,84$ \\
\hline Bid & Proapoptotic (OMM) & $\begin{array}{l}\text { Upon induction of apoptosis translocates to mitochondria and activates } \\
\text { Bax and Bak; induces fusion of mitochondrial cristae; possesses lipid } \\
\text { translocase activity }\end{array}$ & 70,71 \\
\hline $\mathrm{Bcl}-2$ & Antiapoptotic (OMM) & Overexpression causes an increase in size and complexity of mitochondria & 99 \\
\hline VMIA & Antiapoptotic (MITO) & $\begin{array}{l}\text { Viral mitochondria-localized inhibitor of apoptosis, induces mitochondrial } \\
\text { fragmentation }\end{array}$ & 96,97 \\
\hline PB1-F2 & Proapoptotic (MITO) & Viral product of influenza A PB1; induces mitochondrial fragmentation & 81 \\
\hline
\end{tabular}

MITO; unknown submitochondrial localization

chondrial morphology maintenance $(\mathrm{Mmm} 1)^{20-22}$ gene product causes remodeling of the branching, cortical network of mitochondria characteristic of wild-type yeast into one round giant mitochondrion. Mutations of Mdm10, Mdm12 or Mmm1 result also in the inhibition of transport of mitochondria to the buds suggesting that aberrant fission, inhibition of the mitochondrial transport along the cytoskeleton or a combination of both factors cause the phenotype. Mdm10 and Mmm1 are integral proteins of the OMM and possibly play a role in ATP-dependent anchoring of mitochondria to the actin cytoskeleton. ${ }^{23}$ Inhibition of mitochondrial movement along actin filaments observed in $\mathrm{mmm} 1$ and $m d m 10$ mutants may explain the concentration of mitochondria in a small area of the cell, however, formation of a single giant mitochondrion indicates additional functions of Mdm10, Mmm1 and possibly Mdm12 in the regulation of mitochondrial fission. Actually, fragmentation of the mitochondria, not fusion, has been detected in yeast in which the actin cytoskeleton has been disrupted by treatment with latrunculin $A^{23}$ further pointing to a specific fissogenic activity of Mdm10 and Mmm1. There are not known mammalian homologs of the Mdm10, Mmm1 and Mdm 12 proteins, possibly because in mammalian cells mitochondrial motility is a microtubule, not actin, dependent process. ${ }^{24,25} \mathrm{It}$ is worth noting that disruption of microtubules by nocodazole (our unpublished results) or inhibition of mitochondrial movement along microtubules by knockout of mitochondria-specific kinesin motors $\mathrm{KIF}^{2} \mathrm{~B}^{24}$ leads to aberrant distribution, but does not affect the tubular morphology of mitochondria suggesting a less-pronounced morphogenic role of the mitochondrial interactions with the cytoskeleton in mammals than in yeast.

A second class of proteins regulating mitochondrial morphology are members of the dynamin superfamily. Dynamin, a $100 \mathrm{kDa}$ GTPase, is involved in the recycling of synaptic vesicles. ${ }^{26}$ The recent discovery of a number of other proteins sharing a high homology within the dynamin 'GTPase', 'middle' and 'assembly' domains extends the role of dynamin family members to other membrane targets. ${ }^{26,27}$ Yeast Dnm $1^{16,17,28}$ and $M g m 1^{29-30}$ proteins, as well as their homolog in other organisms, ${ }^{31-35}$ regulate mitochondrial 
morphology. Mutations in DNM1, ${ }^{16,17}$ as well as inhibition of a mammalian homolog of Dnm1, dynamin-related protein 1 (Drp1, ${ }^{32}$ also known as DVLP, ${ }^{36} \mathrm{DLP}^{37}$ and Dymple ${ }^{38}$ ), by overexpression of a dominant-negative mutant of Drp1 harboring a mutation in the GTP-binding site (Drp1 ${ }^{\mathrm{K} 38 \mathrm{~A}}$ ), resulted in the formation of extremely interconnected, fused mitochondria. ${ }^{39}$ This phenotype is consistent with an inhibition of Drp1-mediated mitochondrial fission. In addition, Dnm1 and mammalian Drp1 specifically localize to punctuate foci at sites of subsequent mitochondrial fission. ${ }^{16,17,39}$ Colocalization of the Drp1 foci with mitochondrial scission sites has been also demonstrated in Caenorhabditis elegans. ${ }^{33}$ Interestingly, downregulation of Drp1 in $C$. elegans by RNAi inhibited the scission of the OMM, while fission of the IMM was not affected, ${ }^{33}$ suggesting that separate machinery mediates scission of the two mitochondrial membranes. At this point, molecular components governing scission of the IMM are not known and consequently the mechanism(s) synchronizing OMM and IMM fission also remain to be discovered.

Like dynamins, that function as a part of multiprotein complexes, ${ }^{26}$ multiple accessory proteins, in addition to Drp1, appear to be necessary for the proper scission of mitochondria. In yeast, Fis1 (Mdv2) and Mdv1 (Fis2/Gag3/Net2) ${ }^{40-43}$ also are required for fission of mitochondria. Fis1, an integral protein of the OMM, targets Dnm1 to the mitochondria early in the fission pathway, and may also interact with Mdv1, which in turn can bind Dnm1. Mdv1 most probably functions as a molecular adaptor as it interacts with Dnm1 by its C-terminal WD repeats and with Fis 1 by its $\mathrm{N}$-terminal extension region. ${ }^{44}$ Recombinant expression of the isolated WD repeat domain of Mdv1 inhibited mitochondrial fission suggesting a critical role of sequential interactions of Dnm1, Mdv1 and possibly Fis 1 in the severing of the OMM. ${ }^{44}$ Proteins highly homologous to yeast Fis1 are present in Drosophila and in mammals, although it remains unknown whether they participate in the mitochondrial fission complex. ${ }^{42}$

The fact that punctate foci of Drp1 and Mdv1 at mitochondrial fission sites can be detected by the light microscope ${ }^{16,17,37,39,43}$ suggests that multiple copies of Dnm1 and Mdv1 in the fission complex may be necessary to execute mitochondrial scission. Yeast two hybrid and coimmunoprecipitation studies reveal that, in addition to interactions with Fis1 and Mdv1, Dnm1 homo-oligomerizes in vivo. ${ }^{45}$ These results are consistent with those of mammalian Drp1 showing that it resides in the cytosol as a tetramer. ${ }^{46}$ Like dynamin, ${ }^{47}$ Drp1 can assemble into multimeric ring-like structures, in vitro under low salt conditions. ${ }^{39}$ Whether such high molecularweight spiral complexes of Drp1 form in vivo, and their functional importance, is not yet clear. It is currently not known whether Dnm1 mediates mitochondrial scission as a GTP hydrolysis-driven molecular motor, in which case high molecular-weight complexes would cause constriction-inducing scission or, similar to small GTPases, as signaling molecules recruiting other active components to the scission site. The presence of a coiled-coil region, typically a mediator of protein-protein interactions, in Mdv1 and Dnm1 (Drp1) suggests the possible participation of additional proteins in the fission foci. Interestingly, in mammalian cells, in addition to the mitochondrial localization, Drp1-containing structures also colocalize with ER tubules and are adjacent to ER subcom- partments enriched with Rab1. ${ }^{37}$ Furthermore, it has been also shown that Drp1 colocalizes with, and mediates the fission of, peroxisomes ${ }^{48}$ indicating that, like dynamin, Drp1 may mediate scission of multiple membrane targets.

Recently, Rab32, a small GTPase in the Rab protein family, members of which participate in the membrane trafficking at the Golgi apparatus and endosomes, ${ }^{49}$ has been shown to participate in the maintenance of mitochondrial morphology. ${ }^{50}$ Point mutations in the GTP-binding site (T39N) induced formation of abnormally fused mitochondria suggesting that Rab32, like Drp1, may be involved in mitochondrial fission. In spite of the lack of an evident mitochondria-targeting sequence, Rab32 localizes predominantly to mitochondria, as revealed by immunofluorescence analysis. Rab 32 serves also as an A-kinase anchoring protein (AKAP) and sequesters protein kinase $A$ to mitochondria, ${ }^{50}$ although it is not known whether the interaction of Rab32 with PKA contributes to the regulation of mitochondrial morphology.

\section{Regulation of mitochondrial fusion}

Considerable progress has been made recently in the understanding of the molecular mechanism of mitochondrial fusion. The first known mediator of mitochondrial fusion, the Drosophila melanogaster fuzzy onions ( $f z o$ ) gene, encodes a large transmembrane GTPase that does not share detectable homology with the dynamin family of GTPases. ${ }^{51}$ During Drosophila spermatogenesis, mitochondria of early postmeiotic spermatids aggregate and fuse into two giant organelles that wrap around each other. This structure, called a Nebenkern, resembles an onion slice when imaged in cross section by electron microscopy. Fzo1 is upregulated just prior to Nebenkern formation late in meiosis II and disappears when mitochondrial fusion is completed. Mutations in the fzo gene inhibit fusion and formation of the Nebenkern leading to a 'fuzzy onion' morphology. Homologs of the Fzo1 protein are present in yeast ${ }^{52,53}$ and in mammalian cells. ${ }^{54}$ Mutations in yeast $f z 01^{53}$ or knockout of the individual mammalian homologs of Fzo1, mitofusins $1 \& 2$ (Mfn1\&2), ${ }^{55}$ result in the formation of fragmented mitochondria, a phenotype consistent with an inhibition of mitochondrial fusion. Notably, knockout of Mfn1 causes the formation of small round mitochondria, whereas knockout of Mfn2 results in the formation of slightly larger, oval organelles, in addition to the short punctiform mitochondria ${ }^{55}$ suggesting a higher rate of residual fusion in Mfn2-deficient mouse embryonic fibroblasts (MEFs). Striking defects in the mobility of mitochondria in Mfn 1 \& 2- deficient fibroblasts also has been described. In control cells, mitochondria move in the direction of their long axis, consistent with the use of microtubules as tracks, whereas in mutant cells a more random, Brownian-like movement has been detected, especially within the shortest, round mitochondria, indicating that mitochondrial morphology may somehow participate in the regulation of the intracellular mobility of these organelles. Within Mfn2-deficient cells, the slightly elongated, oval mitochondria move in an organized manner resembling control mitochondria, whereas the short, round mitochondria display a random mode of movement. Detailed analysis of mitochondria colabeled with mito-GFP together with the $\Delta \Psi_{\mathrm{m}}$-sensitive mitochondrial probe, Mito- 
tracker Red, in control and Mfn1\&2 knockout MEFs, reveals an increase in mitochondrial heterogeneity, namely an elevation in the number of mitochondria with distinctly lower $\Delta \Psi_{\mathrm{m}}$ in both Mfn1 and Mfn2 mutant cells. Formation of defective mitochondria is most likely the result of an inhibition of a $\Delta \Psi_{\mathrm{m}}$ complementation mechanism that in control cells could result from the frequent fusion and fission of mitochondria. Mfn1 and Mfn2 appear to function differently, in a celltype dependent manner, as revealed by the reduction of the number of the placental trophoblast giant cells, that normally express both Mfn1 and Mfn2, caused by Mfn2 deficiency, but not Mfn1 deficiency in knockout placenta. ${ }^{55}$

Mfn1 and Mfn2 form homotypic and heterotypic complexes. ${ }^{55}$ However, whether different homotypic and heterotypic complexes of Mfns coparticipate in the machinery universal for diverse modes of mitochondrial fusion, or whether they mediate, independent, signal-specific mitochondrial fusion events is not known. Examination of the membrane topology revealed that Fzo1, Mfn2 and likely Mfn1 ${ }^{56,57}$ span the OMM twice, exposing the N-terminal GTPase and C-terminal coiled-coil domains to the cytosol with the short loop between the transmembrane domains located in the intermembrane mitochondrial space. Mutations in the intermembrane space segment of Fzo1 uncovered its importance in the physical coupling of the OMM and IMM, probably through the interactions with a putative IMMassociated component of the fusion machinery. ${ }^{57}$ Yeast harboring mutations in the transmembrane space segment of Fzo1 accumulated fragmented mitochondria, indicating that Fzo1-mediated OMM : IMM interactions are essential for the proper, synchronized fusion of the two mitochondrial membranes. ${ }^{57}$ The N-terminal GTPase and the C-terminal coiledcoil domains of Mfn2 are also necessary for the proper fusion of mitochondria and the C-terminal and middle coiled-coil domains are required for the proper mitochondrial localization of Mfn2. ${ }^{56}$ Recombinant expression of GFP-tagged Mfn2 revealed that Mfn2 can concentrate in punctate foci on mitochondria at sites of fusion/fission. ${ }^{58}$ Truncation of the Cterminal coiled-coil domain (GFP-Mfn2 ${ }^{1-703}$ ) alters the submitochondrial localization of GFP-Mfn2, shifting it from partly circumscribing the OMM and partly localized to punctuate submitochondrial foci to completely circumscribing the OMM (our unpublished observations). Expression of GFP-Mfn2 ${ }^{1-703}$ in Mfn2 knockout MEFs does not reconstitute the tubular, elongated morphology of mitochondria, as overexpression of WT GFP-Mfn2 does (unpublished results). Thus, the Cterminal coiled-coil domain is required for fusion activity and also for submitochondrial localization in the foci. Mutation of the GTP-binding site in the GTPase domain also results in the inactivation of the Mfns. ${ }^{55}$

Recently, using a genetic screen of mutant yeast, Sesaki and Jensen ${ }^{59}$ found a gene coding a novel protein necessary for mitochondrial fusion, UGO1. Ugo1 localizes to the OMM with an $\mathrm{N}$-terminal domain facing the cytosol and the $\mathrm{C}$ terminus localized in the intermembrane space. It is not known whether its fusogenic activity requires interactions with Fzo1 or whether several independent stages of mitochondrial fusion exist.

Another mediator of mitochondrial fusion, the large dynamin-like GTPase, Mgm1, may participate in the fusion and remodeling of the IMM. It has been demonstrated that Mgm1 localizes to the external side of the IMM and that the loss of MGM1 function leads to the fragmentation of the mitochondrial reticulum. ${ }^{30}$ However, a specific role of Mgm1 in the fusion of the IMM remains controversial, as another report indicates that Mgm1 behaves as an integral protein of the $\mathrm{OMM}^{29}$ Despite the phenotypic similarities between $\mathrm{mgm} 1$ and fzo1 mutant cells, loss of MGM1 function does not affect fusion of mitochondria during yeast mating. ${ }^{30}$ Mammalian homologs of Mgm1 have been characterized. ${ }^{31,34,35,60}$ In humans, mutations in the Mgm1 homolog have been detected in patients with dominant optic atrophy (mammalian homologues of Mgm1 are thus called OPA1), a neuropathy resulting from the loss of retinal ganglion cells. ${ }^{31,34}$ Human OPA1 is tightly attached to the outer leaflet of the IMM, close to the cristae, as revealed by biochemical and immunoelectron microscopy analysis. ${ }^{60}$ Modifications of the mitochondrial morphology in cells expressing different OPA1 constructs were detected. Notably, expression of GFP-tagged full-length OPA1 induced the formation of a filamentous network of mitochondria, whereas the N-terminal, 120 amino-acid-long mitochondrial targeting region tagged with GFP did not affect mitochondrial morphology. ${ }^{60}$

Experiments using yeast mutants lacking mitochondrial fusion, fission and double mutants deficient in both of these processes, ${ }^{16,17}$ established the critical role of balancing of fusion and fission in the regulation of mitochondrial shape and connectivity in yeast. The discovery and characterization of the various proteins governing mitochondrial membrane remodeling, described above, create new, exciting challenges for mitochondrial research. How are the actions of those separate elements, likely receiving signals from extramitochondrial compartments of the cells as well as from the mitochondria, integrated into a synchronized process? Moreover, mammalian proteins that, in addition to Drp1 and Rab32, mediate scission of mitochondria remain to be discovered. These and many more questions are waiting to be answered in this fascinating new area of cell biology.

\section{Mitochondrial remodeling and fragmentation during apoptosis}

Recent developments in apoptosis research have uncovered a central role of mitochondria. Initially, mitochondrial participation in apoptosis was connected with the collapse of $\Delta \Psi_{\mathrm{m}}$ that was considered a point of no return in the death cascade. $^{61}$ The hypothesis that during early stages of apoptosis several proteins such as cytochrome $c$ and smac/ Diablo are released from the mitochondrial intermembrane space to the cytosol, where they bind to specific cytosolic targets and subsequently activate the caspase-activating pathways, also suggested that mitochondria may serve as a decision maker regulating the viability of cells. Cytochrome $c$ is released from mitochondria upon induction of apoptosis and forms the 'apoptosome' in the cytosol together with the adaptor protein Apaf-1, to activate procaspase- 9 and subsequently the effector caspases-3 and -7 . The mechanism of OMM permeabilization that releases cytochrome $c$ and what kind, if any, of mitochondrial priming that has to occur before 
cytochrome $c$ release is not well understood. Pro- and antiapoptotic proteins from the $\mathrm{Bcl}-2$ protein family seem to be central regulators of the OMM permeability. Members of the $\mathrm{BH} 3$ only subset of the Bcl-2 family, including Bad, Bid, Bim, and others transduce prodeath signals from different compartments of the cell and initiate OMM permeabilization, either by activation of the proapoptotic members, Bax and Bak (Bax-like proteins), or inactivation of the antiapoptotic members, $\mathrm{Bcl}-2$ and $\mathrm{Bcl}-\mathrm{X}_{\mathrm{L}}{ }^{62}$ Multidomain proapoptotic members of the Bcl-2 family, Bax and Bak, are indispensable effectors of the apoptotic pathway. ${ }^{63}$ Multiple models of Baxmediated permeabilization of the OMM have been proposed, including interaction of Bax with components of the PTP, the voltage-dependent anion-selective channel (VDAC) ${ }^{64}$ or $\mathrm{ANT}^{65}$ or, based on the structural similarity to certain poreforming bacterial toxins and supported by in vitro channel-like activity of some Bcl-2 family members, direct pore formation. ${ }^{66,67}$ Interestingly, contrary to reports describing a preservation of the structure of mitochondria challenged with recombinant Bid or Bax, ${ }^{68,69}$ it has recently been suggested that tBid might mediate, independent of its interactions with Bax-like proteins, a remodeling of the mitochondrial cristae that facilitates an early, prerelease relocation of cytochrome $c$ from cristae stores and mobilization in the intermembrane space. In healthy cells, most of the cytochrome $c$ localizes in the mitochondrial cristae, separated from the intermembrane space by a narrow cristae junction (discussed in Frey and Mannella ${ }^{4}$ ) that can be somehow opened by tBid ${ }^{70}$ (Table 2). Although the mechanism of tBid-mediated opening of the cristae junctions is unknown, electron microscopy tomography of tBid-treated mitochondria revealed an increase of the intracristae connectivity and formation of a more highly interconnected cristae network suggesting the activation of IMM fusion. Remodeling of the mitochondrial cristae could also be seen in vivo in apoptotic cells, ${ }^{70}$ suggesting the possibility that regulated changes of the mitochondrial morphology may generally occur early in the apoptotic process. It has been reported that tBid has intrinsic lipid translocase activity, ${ }^{71}$ and can directly affect the curvature of the liposomes. ${ }^{72}$ One may speculate that tBid may participate in a lipid-modifying multiprotein complex, likely localized at the OMM : IMM contact sites that regulate sequential changes of the mitochondrial ultrastructure. There is very little information on the factors regulating the structure of mitochondrial cristae. It has been suggested that two nonessential subunits ( $e$ and $g$ ) of yeast ATP synthase may be responsible for cristae formation through coiled-coil-mediated ATP synthase dimerisation and oligomer formation followed by a zipper-like mechanism-induced invagination of the IMM. ${ }^{73}$ However, it is not known whether, in addition to cristae formation, dynamics of preformed cristae are also regulated by subunits of ATP synthase. Another factor, Mgm1/OPA1, as discussed previously, may participate in cristae remodeling. Experimental downregulation of OPA1 using siRNA led to alterations in the morphology of the cristae and formation of unusual vesicle-like structures with an increased diameter somewhat resembling those induced by $t B i d$. Alterations of mitochondrial structure caused by OPA1 depletion are associated with the release of cytochrome $c$ from mitochondria to the cytosol and subsequent induction of apoptotic cell death. ${ }^{74}$ Notably, in addition to the remodeling of mitochondrial cristae, depletion of OPA1 results in the fragmentation of the mitochondrial network, similar in morphology to that observed in $\Delta m g m 1$ yeast. It is not clear whether OPA1 downregulation-induced OMM permeabilization occurs due to cristae remodeling or due to the activation of mitochondrial fragmentation.

Mitochondrial fragmentation has been described in connection with many modes of apoptosis (Figure 3). ${ }^{58,74-82}$ Ultrastructural analysis of Colo-205 cells treated with a tyrosine kinase inhibitor, Herbimycin A, to induce apoptosis revealed a distinct increase in the number of mitochondria consistent with mitochondrial fragmentation. Fragmentation of mitochondria occurred in parallel with the formation of apoptotic bodies, an increase in the number of TUNELpositive nuclei and cleavage of the caspase substrate PARP. ${ }^{80}$ It is not clear whether mitochondrial fragmentation observed in Herbimycin A-treated cells is a nonspecific epiphenomenona, or whether it participates directly in the transduction of an apoptotic signal. However, the potential role of mitochondrial fragmentation and dynamin family proteins participating in the regulation of mitochondrial morphology, in the control of apoptosis was described by Frank et al. ${ }^{77}$ When the morphology of mitochondria stained with fluorescent probes was analyzed over time by confocal microscopy, a transition from a mitochondrial network into vesicular punctiform mitochondria was invariably detected in cells treated with various apoptotic stimuli, beginning at the very early stages of the cell death cascade. Changes in mitochondrial morphology were associated with a distinct increase in the number of mitochondria suggesting the participation of mitochondrial fission machinery. Apoptotic fragmentation of mitochondria was not affected by caspase inhibitors but, interestingly, was inhibited by ectopic expression of the dominant-negative mutant of Drp1, Drp1 ${ }^{\mathrm{K} 38 \mathrm{~A}}$. Moreover, Drp $1^{\mathrm{K} 38 \mathrm{~A}}$ not only inhibited apoptotic fragmentation of mitochondria, but also inhibited or delayed cytochrome $c$ release, the decrease in $\Delta \Psi_{\mathrm{m}}$ and fragmentation of nuclear DNA, all markers of apoptosis. An inhibitory effect of a similar dominant-negative mutant of Drp1, Drp $1^{\mathrm{K} 38 \mathrm{E}}$, on mitochondrial fragmentation and cell death induced by p20, a caspase- a

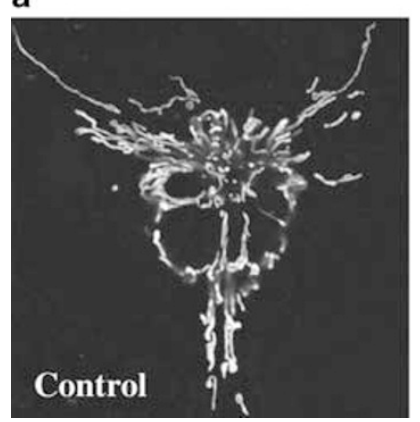

b

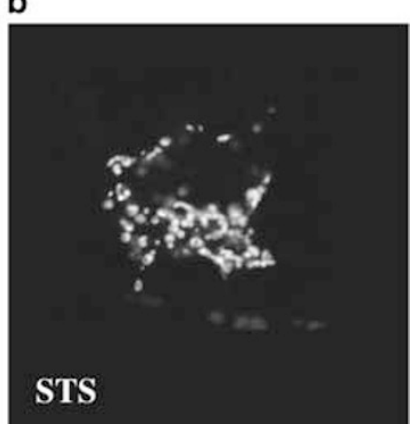

Figure 3 Mitochondrial fragmentation during apoptosis. Disintegration of the tubular mitochondrial network and formation of punctiform, fragmented mitochondria occur early during apoptosis induced by variety of the stimuli (see details in the text). Examples of control (a) and STS-treated (b) Cos-7 cells are shown. Cells were transfected with mito-YFP $(\mathbf{a}, \mathbf{b})$ and at $12 \mathrm{~h}$ after transfection were untreated (a) or treated with $1 \mu \mathrm{M}$ STS (b) for $180 \mathrm{~min}$ and imaged using confocal microscopy 
8 cleavage product of the ER integral protein BAP31, has been also reported. ${ }^{82}$ Formation of p20 increased $\mathrm{Ca}^{2+}$ transfer between the ER and mitochondria that somehow affected the mitochondrial fission machinery resulting in the fragmented phenotype of the mitochondria. Overexpression of Drp1 ${ }^{\mathrm{K} 38 \mathrm{E}}$ was sufficient to distinctly inhibit mitochondrial permeabilization assessed by cytochrome $c$ release, as well as caspase activation. ${ }^{82}$

Overexpression of Bax, but not a mutant Bax lacking the $\mathrm{BH} 3$ domain, distinctly amplified mitochondrial fragmentation induced by the proapoptotic agent staurosporine (STS), further connecting mitochondrial fragmentation to the apoptotic process. ${ }^{77}$ It also has been reported that in HeLa cells overexpressing Bax and cultured in the presence of the caspase inhibitor z-VADfmk, a collapse of the mitochondrial network and formation of punctiform mitochondria followed by perinuclear clustering of fragmented organelles can occur spontaneously without additional apoptotic stimuli, ${ }^{75}$ suggesting a direct role of Bax and, potentially, other members of Bcl2 family in the fragmentation of mitochondrial network.

How Bax induces mitochondrial fragmentation remains to be established. However, it has been shown that upon induction of apoptosis Bax translocates to the OMM ${ }^{83}$ and, almost instantly after translocation, concentrates into submitochondrial punctate foci. ${ }^{84}$ In addition, Bak, the closest Bax homolog from the Bcl-2 family, colocalizes with Bax in these foci. ${ }^{84}$ In STS-treated Cos7 and HeLa cells, Bax/Bak foci formation is associated with a disruption of the mitochondrial network ${ }^{58}$ and, moreover, Bax foci frequently appear at the sites of mitochondrial scission (Figure 4). Fragmentation of mitochondria occurring after Bax mitochondrial translocation and formation of submitochondrial foci has been also described to occur in STS-treated cardiomyocytes. ${ }^{76}$ Drp1 and Mfn2 colocalize with Bax in foci in different cell types treated with various inducers of stress-dependent apoptosis $(\text { Figure } 5)^{58}$ suggesting that Bax may be targeting subdomains of mitochondria that are most prone to dynamic membrane rearrangements or may somehow interfere with the activity of the mitochondrial fusion/fission machinery. The dominant-negative inhibitor of Drp1, Drp ${ }^{\mathrm{K} 38 \mathrm{~A}}$, does not affect the dynamics of Bax translocation and foci formation, but inhibits Bax-induced mitochondrial fragmentation. As docu-
CFP-Bax

a mito-YFP

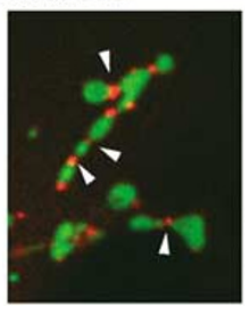

mito-YFP

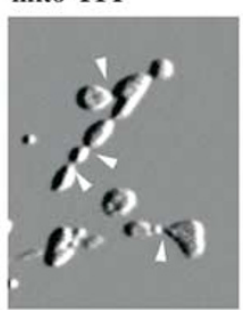

b

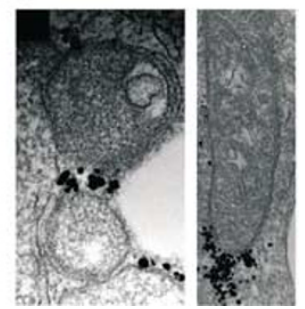

Figure 4 Bax coalesces in foci localized at mitochondrial scission sites (a) Cos7 cells co-transfected with CFP-Bax (red) and mito-YFP (green) were treated with STS and analyzed by confocal microscopy. The image of mitochondria (a left panel) was processed using Adobe Photoshop. (b) Cos7 cells were transfected with GFP-Bax, treated with STS and processed for immunoelectron microscopy. For experimental details see Karbowski et al..$^{58}$ Note the localization of CFP-Bax at tips and at mitochondrial constriction sites (arrowheads in a)
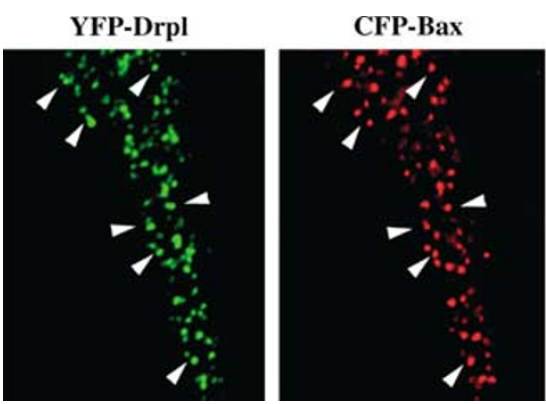

Mitochondria



Figure 5 In apoptotic cells Bax colocalizes with Drp1. HeLa cells were cotransfected with CFP-Bax (red), YFP-Drp1 (green) and mito-DsRed2, then treated with STS and analyzed by confocal microscopy (for experimental details see Karbowski et $a^{58}$ ). The image of mitochondria (right panel) was processed using Adobe Photoshop. Note that most of the Bax clusters precisely colocalize with Drp1positive subdomains of the mitochondria (examples are marked by arrowheads)

mented by confocal and immunoelectron microscopy, even in the absence of active Drp1, deformations of the OMM resembling mitochondrial constriction sites are often present at the sites of Bax-foci formation, ${ }^{58}$ suggesting the participation of additional factors, or that Bax itself possesses membrane-deforming capability. Both possibilities are supported by independent sets of data. Yeast two hybrid screens using C-terminal truncated Bax as the bait revealed strong interactions of Bax with a member of the endophilin protein family called Bax-interacting factor 1 (Bif1), ${ }^{85}$ SH3GLB1, ${ }^{86}$ or endophilin $B 1^{87}$ (for simplicity, the name Bif1 will be used). Binding of Bif1 and Bax was also recapitulated by coimmunoprecipitation experiments, and appeared to be specifically enhanced by the proapoptotic conditions such as interleukin-3 withdrawal $^{85}$ or treatment with an epothilone B analog (BMS247550). ${ }^{88}$ It is not clear how Bif1 affects Bax, but there are interesting indications suggesting that Bif1 has membranedeforming activity. Members of the endophilin protein family participate in the regulation of membrane curvature and dynamics in multiple cellular processes including endocytosis of synaptic vesicles. ${ }^{89,90}$ Interestingly, Bif1, as well as endophilin 1, can directly tubulate liposomes. ${ }^{87}$ Bif1-mediated OMM deformations may somehow facilitate Bax interactions with the OMM and the subsequent Bax activation of apoptosis. Indeed, recombinant expression of Bif1 in FL5.12 cells facilitates interleukin-3 withdrawal-induced exposure of the Bax N-terminal epitope $(6 \mathrm{~A} 7)^{85}$ that is hidden in healthy cells and becomes exposed upon apoptotic activation of Bax. ${ }^{91}$ Importantly, Bax-mediated liposome permeabilization occurs through a mechanism sensitive to intrinsic membrane curvature. $^{92}$ Bif1 binds Bax by its $\mathrm{N}$-terminal eight amino acids, ${ }^{86}$ suggesting the possibility of coordinated interactions with additional protein proline-reach domains mediated by the Bif1 C-terminal SH3 domain, and with Bax mediated by the $\mathrm{N}$ terminus. Endophilin family proteins have been shown to bind dynamin family members via the C-terminal SH3 domain. ${ }^{26}$ Our analysis of the amino-acid sequence of Drp1 reveals that the proline-rich region and, the PXXP motif in amino acids 610-618 (PIPIMPASP), a potential SH3-binding site, may bind the endophilin, Bif1. Interestingly, homologous SH3 binding motifs are also present in Mfn2 (amino acids 523-526, 
PLLP, and amino acids 595-609, PIPLTPANPSMPPLP), suggesting that Drp1 and Mfn2 may have similar binding partners. Overexpression or microinjection of oligopeptides containing one or two PXXP motifs inhibited the binding of dynamin to the protein regulators of membrane fission, amphiphysin and PLC. ${ }^{26}$ Bif1 also binds another endophilin, SH3GLB2 ${ }^{86}$ suggesting a variety of potential Bax-interacting proteins. Another potential candidate for the Bif1 SH3 domainbinding partner is the mitochondrial synaptojanin $2 \mathrm{~A}$. Recombinant overexpression of mitochondrial targeted synaptojanin $2 \mathrm{~A}$ was shown to have effects on the gross morphology of mitochondria. $^{93}$

Treatment with the $\mathrm{Ca}^{2+}$ ionophore, A23187, also induces fragmentation of the mitochondrial network in myoblasts, astrocytes $^{94}$ and HeLa cells that occurs in the absence of Bax translocation and is not directly followed by apoptotic cell death (our unpublished results), suggesting that neither Baxfoci formation nor apoptosis are necessary triggers of mitochondrial fragmentation. Reports from the Rizzuto's group ${ }^{78,79}$ suggest an important role of $\mathrm{Ca}^{2+}$ in the regulation of the mitochondrial connectivity during apoptosis and quite likely during other physiological conditions. They show that an early rupture of the mitochondrial network occurs in HeLa cells treated with the Bcl-2-sensitive apoptotic stimulus, ceramide. ${ }^{78,79}$ Ceramide causes an early increase in the level of cytosolic $\mathrm{Ca}^{2+}$ that is associated with fragmentation of the mitochondrial network. Conditions reducing the cytoplasmic $\mathrm{Ca}^{2+}$ increase were sufficient to prevent mitochondrial fragmentation and also inhibited ceramide-mediated cell death. However, whether the mitochondrial fragmentation is temporally associated with Bax translocation and foci formation in this system is not known. Experiments with digitoninpermeabilized cells incubated with caspase-8-cleaved Bid revealed an important role of tBid in the regulation of $\mathrm{Ca}^{2+}$ transport at focal contact sites between the ER and the mitochondria. ${ }^{95}$ As the proapoptotic effect of $t B i d$ relies on the presence of $\mathrm{Bax}$ and $\mathrm{Bak},{ }^{63}$ one may speculate that tBidmediated Bax activation may lead to local Bax foci that increase the permeability of the OMM for $\mathrm{Ca}^{2+}$ that in turn lead to the activation of the mitochondrial fission machinery localized in the same subcompartments of the OMM. Interestingly, recombinant expression of VDAC, that can amplify the transfer of $\mathrm{Ca}^{2+}$ from ER to mitochondria, potentiates the fragmentation of the mitochondrial network induced by ceramide, ${ }^{79}$ confirming the potential role of $\mathrm{Ca}^{2+}$ microdomains in the regulation of mitochondrial connectivity during apoptosis. Dynamin-mediated endocytosis is regulated by the local concentration of $\mathrm{Ca}^{2+}$ and, as discussed above, direct effects of $\mathrm{Ca}^{2+}$ on mitochondrial fission have been confirmed by the effects of calcium ionophores and caffeine (Duncan et $a^{\rho^{4}}$ and our unpublished results) suggesting the possibility that $\mathrm{Ca}^{2+}$ may directly regulate Drp1 and other proteins participating in mitochondrial fission foci.

Although the mechanism and role of the mitochondrial fragmentation in apoptosis remains to be confirmed, its universal occurrence was further suggested by recent reports connecting two different viral protein modulators of apoptosis with the morphology of mitochondria. Screening for alternative-reading frames encoded in the influenza A virus (PR8) revealed the presence of an abundant, immunogenic, amino-acid protein (PB1-F2) coded by the +1 reading frame of PB1. Recombinant expression of the synthetic version of PB1-F2 induced apoptosis, whereas targeted mutations that interfere with PB1-F2 repressed apoptosis induced by PR8 infection. ${ }^{81}$ As shown by indirect immunofluorescence of MDBK cells infected with PR8 or transfected with PB1-F2 CDNA, PB1-F2 localizes predominantly to mitochondria. Notably, expression of PB1-F2 in cells induced the rounding of mitochondria consistent with fragmentation associated with a decrease in the $\Delta \Psi_{\mathrm{m}}$. Alterations of mitochondrial morphology and function were also observed in cells injected with synthetic peptides corresponding to the fulllength PB1-F2. How PB1-F2 induces mitochondrial fragmentation and activates apoptosis is not known. However, the toxic effect of PB1-F2, including the fragmentation of mitochondria and the release of cytochrome $c$, can be suppressed by overexpression of $\mathrm{Bcl}-2$ suggesting the participation of the members of $\mathrm{Bcl}-2$ protein family in the action of PB1-F2.

The cytomegalovirus UL37 gene product (also called viral mitochondrion-localized inhibitor of apoptosis, vMIA) has been reported to have a potent antiapoptotic activity. In a similar manner to that of PB1-F2, vMIA localizes to and induces morphological alterations of mitochondria consistent with fragmentation, confirmed by immunocytochemistry and mitochondrial sedimentation assays, but unlike PB1-F2 that induces cytochrome $c$ release, VMIA protects cells against apoptosis. ${ }^{96,97}$ vMIA inhibits Fas-mediated apoptosis downstream of Bid cleavage, but upstream of cytochrome $c$ release, ${ }^{97}$ raising the possibility that Bax-like proteins may be its target. Interestingly, the punctate staining of the subcellular VMIA localizations follows a mitochondrial pattern but does not completely colocalize with Mitotracker-stained mitochondria (see Figure $2 \mathrm{~B}$ in McCormick et al. ${ }^{96}$ ). Moreover, it has been shown that VMIA resides not only in the mitochondria, but also in the ER and Golgi apparatus. ${ }^{98} \mathrm{~A}$ subpopulation of Drp1 has been also found to colocalize with ER and mitochondria. One may speculate that VMIA may block apoptosis by interfering with Drp1 or another factor from the mitochondrial fission machinery at a step downstream of Bax activation, foci formation and fragmentation of the mitochondrial network but upstream of mitochondrial permeabilization. Further experiments are clearly needed to establish whether there is a firm correlation between antiapoptotic activity and mitochondrial morphology regulatory functions of vMIA.

Finally, it has been recently reported that overexpression of the antiapoptotic $\mathrm{Bcl}-2$ protein can cause increases in the volume and structural complexity of the mitochondria, without affecting $\Delta \Psi_{\mathrm{m}}, \Delta \mathrm{pH}$ or intramitochondrial $\left\{\mathrm{K}^{+}\right\}$in $\mathrm{PC} 12$ and GT1-7 neural cell lines. ${ }^{99}$ Although no microscopic data were presented, flow cytometric particle sizing and light-scattering determinations performed together with an analysis of mitochondrial uptake of $\Delta \Psi_{\mathrm{m}^{-}}$and $\mathrm{K}^{+}$-sensitive fluorescent probes, of the mitochondria isolated from control and $\mathrm{Bcl}-2$ overexpressing cells, revealed an increased membrane area and structural complexity in the mitochondria isolated from Bcl-2 overexpressing cells. Thus, in addition to or by interacting with Bid and Bax, other members of the Bcl-2 
protein family appear to participate in the regulation of mitochondrial morphology.

Although mitochondrial fragmentation can occur without activation of apoptosis, apoptosis may not be able to occur without activation of the mitochondrial scission machinery. The findings that dominant-negative mutant of Drp1 blocks mitochondrial fission during apoptosis and inhibits cell death, together with the opposite tendency of fragmentation and a decrease in the complexity of mitochondria, that can be detected during apoptosis induced by variety of treatments and upon Bax overexpression, supports the possibility that conditions favoring mitochondrial connectivity may be beneficial for cell survival, not only in normal growth conditions, as described in the first part of this review, but also when cells are challenged with apoptotic stimuli. However, clearly, additional experiments are necessary to determine the importance and role of mitochondrial network maintenance in the regulation of apoptotic cell death.

\section{References}

1. Bereiter-Hahn J and Voth M (1994) Dynamics of mitochondria in living cells, shape changes, dislocations, fusion, and fission of mitochondria. Microsc. Res. Tech. 27: 198-219

2. Chen LB (1988) Mitochondrial membrane potential in living cells. Annu. Rev. Cell. Biol. 4: 155-181

3. Rizzuto R, Simpson AW, Brini M and Pozzan T (1992) Rapid changes of mitochondrial $\mathrm{Ca}^{2+}$ revealed by specifically targeted recombinant aequorin. Nature 358: 325-327

4. Frey TG and Mannella CA (2000) The internal structure of mitochondria. Trends Biochem. Sci. 25: 319-324

5. Rizzuto R, Pinton P, Carrington W, Fay FS, Fogarty KE, Lifshitz LM, Tuft RA and Pozzan T (1998) Close contacts with the endoplasmic reticulum as determinants of mitochondrial $\mathrm{Ca}^{2+}$ responses. Science 280: 1763-1766

6. De Giorgi $F$, Lartigue $L$ and Ichas $F(2000)$ Electrical coupling and plasticity of the mitochondrial network. Cell Calcium 28: 365-370

7. Skulachev VP (2001) Mitochondrial filaments and clusters as intracellular power-transmitting cables. Trends Biochem. Sci. 26: 23-29

8. Nakada K, Inoue K, Ono T, Isobe K, Ogura A, Goto YI, Nonaka I and Hayashi JI (2001) Inter-mitochondrial complementation: mitochondria-specific system preventing mice from expression of disease phenotypes by mutant mtDNA. Nat. Med. 7: 934-940

9. Egner A, Jakobs S and Hell SW (2002) Fast $100-\mathrm{nm}$ resolution threedimensional microscope reveals structural plasticity of mitochondria in live yeast. Proc. Natl. Acad. Sci. USA 99: 3370-3375

10. Hoffmann HP and Avers CJ (1973) Mitochondrion of yeast: ultrastructural evidence for one giant, branched organelle per cell. Science 181: 749-751

11. Nunnari J, Marshall W, Straight A, Murray A, Sedat J and Walter P (1997) Mitochondrial transmission during mating in Saccharomyces cerevisiae is determined by mitochondrial fusion and fission and the intramitochondrial segregation of mitochondrial DNA. Mol. Biol. Cell. 8: 1233-1242

12. Collins TJ, Berridge MJ, Lipp P and Bootman MD (2002) Mitochondria are morphologically and functionally heterogeneous within cells. EMBO J. 21: 1616-1627

13. Park MK, Ashby MC, Erdemli G, Petersen $\mathrm{OH}$ and Tepikin AV (2001) Perinuclear, perigranular and sub-plasmalemmal mitochondria have distinct functions in the regulation of cellular calcium transport. EMBO J. 20: 18631874

14. Gilkerson RW, Margineantu DH, Capaldi RA and Selker JML (2000) Mitochondrial DNA depletion causes morphological changes in the mitochondrial reticulum of cultured human cells. FEBS Lett. 474: 1-4

15. Legros F, Lombes A, Frachon $P$ and Rojo $M(2002)$ Mitochondrial fusion in human cells is efficient, requires the inner membrane potential, and is mediated by mitofusins. Mol. Biol. Cell. 13: 4343-4354
16. Bleazard W, McCaffery JM, King EJ, Bale S, Mozdy A, Tieu Q, Nunnari J and Shaw JM (1999) The dynamin-related GTPase Dnm1 regulates mitochondrial fission in yeast. Nat. Cell. Biol. 1: 298-304

17. Sesaki $H$ and Jensen RE (1999) Division versus fusion: Dnm1p and Fzo1p antagonistically regulate mitochondrial shape. J. Cell. Biol. 147: 699-706

18. Yaffe MP (1999) The machinery of mitochondrial inheritance and behavior. Science 283: 1493-1497

19. Dimmer KS, Fritz S, Fuchs F, Messerschmitt M, Weinbach N, Neupert W and Westermann B. (2002) Genetic basis of mitochondrial function and morphology in Saccharomyces cerevisiae. Mol. Biol. Cell., 13: 847-853

20. Sogo LF and Yaffe MP (1994) Regulation of mitochondrial morphology and inheritance by Mdm10p, a protein of the mitochondrial outer membrane. J. Cell. Biol. 126: 1361-1373

21. Burgess SM, Delannoy M and Jensen RE (1994) MMM1 encodes a mitochondrial outer membrane protein essential for establishing and maintaining the structure of yeast mitochondria. J. Cell. Biol., 126: 1375-1391

22. Berger KH, Sogo LF and Yaffe MP (1997) Mdm12p, a component required for mitochondrial inheritance that is conserved between budding and fission yeast. J. Cell. Biol., 136: 545-553

23. Boldogh I, Vojtov N, Karmon S and Pon LA (1998) Interaction between mitochondria and the actin cytoskeleton in budding yeast requires two integral mitochondrial outer membrane proteins, Mmm1p and Mdm10p. J. Cell Biol., 141: 1371-1381

24. Tanaka Y, Kanai Y, Okada Y, Nonaka S, Takeda S, Harada A and Hirokawa N (1998) Targeted disruption of mouse conventional kinesin heavy chain, kif5B, results in abnormal perinuclear clustering of mitochondria. Cell 93 : $1147-1158$

25. Nangaku M, Sato-Yoshitake R, Okada Y, Noda Y, Takemura R, Yamazaki $H$ and Hirokawa N (1994) KIF1B, a novel microtubule plus enddirected monomeric motor protein for transport of mitochondria. Cell 79 : 1209-1220

26. Hinshaw JE (2000) Dynamin and its role in membrane fission. Annu. Rev. Cell Dev. Biol. 16: 483-519

27. van der Bliek AM (1999) Functional diversity in the dynamin family. Trends Cell. Biol. 9: 96-102

28. Otsuga D, Keegan BR, Brisch E, Thatcher JW, Hermann GJ, Bleazard W and Shaw JM. (1998) The dynamin-related GTPase, Dnm1p, controls mitochondrial morphology in yeast. J. Cell. Biol., 143: 333-349

29. Shepard KA, Yaffe MP (1999) The yeast dynamin-like protein, Mgm1p, functions on the mitochondrial outer membrane to mediate mitochondrial inheritance. J. Cell Biol., 144: 711-720

30. Wong ED, Wagner JA, Gorsich SW, McCaffery JM, Shaw JM and Nunnari J. (2000) The dynamin-related GTPase, Mgm1p, is an intermembrane space protein required for maintenance of fusion competent mitochondria. J. Cell Biol., 151: 341-352

31. Delettre C, Lenaers G, Griffoin JM, Gigarel N, Lorenzo C, Belenguer P, Pelloquin L, Grosgeorge J, Turc-Carel C, Perret E, Astarie-Dequeker C, Lasquellec L, Arnaud B, Ducommun B, Kaplan J and Hamel CP (2000) Nuclear gene OPA1, encoding a mitochondrial dynamin-related protein, is mutated in dominant optic atrophy. Nat. Genet. 26: 207-210

32. Imoto M, Tachibana I and Urrutia R (1998) Identification and functional characterization of a novel human protein highly related to the yeast dynaminlike GTPase Vps1p. J. Cell. Sci. 111 (Part 10): 1341-1349

33. Labrousse AM, Zappaterra MD, Rube DA and van der Bliek AM (1999) C. elegans dynamin-related protein DRP-1 controls severing of the mitochondrial outer membrane. Mol. Cell 4: 815-826

34. Alexander C, Votruba M, Pesch UE, Thiselton DL, Mayer S, Moore A Rodriguez M, Kellner U, Leo-Kottler B, Auburger G, Bhattacharya SS and Wissinger B (2000) OPA1, encoding a dynamin-related GTPase, is mutated in autosomal dominant optic atrophy linked to chromosome 3q28. Nat. Genet. 26 : $211-215$

35. Misaka T, Miyashita T and Kubo Y (2002) Primary structure of a dynaminrelated mouse mitochondrial GTPase and its distribution in brain, subcellular localization, and effect on mitochondrial morphology. J. Biol. Chem., 277: 15834-15842

36. Shin HW, Shinotsuka C, Torii S, Murakami K and Nakayama K (1997) Identification and subcellular localization of a novel mammalian dynaminrelated protein homologous to yeast Vps1p and Dnm1p. J. Biochem. (Tokyo) 122: $525-530$ 
37. Yoon Y, Pitts KR, Dahan S and McNiven MA. (1998) A novel dynamin-like protein associates with cytoplasmic vesicles and tubules of the endoplasmic reticulum in mammalian cells. J. Cell. Biol., 140: 779-793

38. Kamimoto $T$, Nagai $Y$, Onogi $H$, Muro $Y$, Wakabayashi $T$ and Hagiwara $M$ (1998) Dymple, a novel dynamin-like high molecular weight GTPase lacking a proline-rich carboxyl-terminal domain in mammalian cells. J. Biol. Chem. 273: 1044-1051

39. Smirnova E, Griparic L, Shurland D-L and van der Bliek AM. (2001) Dynaminrelated Protein Drp1 is required for mitochondrial division in mammalian cells. Mol. Biol. Cell., 12: 2245-2256

40. Cerveny KL, McCaffery JM and Jensen RE (2001) Division of mitochondria requires a novel DNM1-interacting protein, Net2p. Mol. Biol. Cell., 12: 309-321

41. Fekkes P, Shepard KA and Yaffe MP (2000) Gag3p, an outer membrane protein required for fission of mitochondrial tubules. J. Cell. Biol., 151: 333-340

42. Mozdy AD, McCaffery JM and Shaw JM. (2000) Dnm1p GTPase-mediated mitochondrial fission is a multi-step process requiring the novel integral membrane component Fis1p. J. Cell. Biol. 151: 367-380

43. Tieu $Q$ and Nunnari J. (2000) Mdv1p is a WD repeat protein that interacts with the dynamin-related GTPase, Dnm1p, to trigger mitochondrial division. J. Cell. Biol. 151: 353-366

44. Tieu Q, Okreglak V, Naylor K and Nunnari J (2002) The WD repeat protein, Mdv1p, functions as a molecular adaptor by interacting with Dnm1p and Fis $1 p$ during mitochondrial fission. J. Cell. Biol. 158: 445-452

45. Fukushima NH, Brisch E, Keegan BR, Bleazard W and Shaw JM. (2001) The GTPase effector domain sequence of the Dnm1p GTPase regulates selfassembly and controls a rate-limiting step in mitochondrial fission. Mol. Biol. Cell. 12: 2756-2766

46. Shin H-W, Takatsu H, Mukai H, Munekata E, Murakami K, Nakayama K (1999) Intermolecular and interdomain interactions of a dynamin-related GTP-binding protein, Dnm1p/Vps1p-like protein. J. Biol. Chem. 274: 2780-2785

47. Hinshaw JE and Schmid SL (1995) Dynamin self-assembles into rings suggesting a mechanism for coated vesicle budding. Nature 374: 190-192

48. Koch A, Thiemann M, Grabenbauer M, Yoon Y, McNiven MA and Schrader M (2002) The dynamin-like protein DLP1 is involved in peroxisomal fission. J. Biol. Chem. 278: 8597-8605

49. Takai Y, Sasaki T and Matozaki T (2001) Small GTP-binding proteins. Physiol. Rev. 81: 153-208

50. Alto NM, Soderling $J$ and Scott JD (2002) Rab32 is an A-kinase anchoring protein and participates in mitochondrial dynamics. J. Cell Biol. 158: 659-668

51. Hales KG and Fuller MT (1997) Developmentally regulated mitochondrial fusion mediated by a conserved, novel, predicted GTPase. Cell. 90: 121-129

52. Rapaport D, Brunner M, Neupert W and Westermann B (1998) Fzo1p is a mitochondrial outer membrane protein essential for the biogenesis of functional mitochondria in Saccharomyces cerevisiae. J. Biol. Chem. 273: 20150-20155

53. Hermann GJ, Thatcher JW, Mills JP, Hales KG, Fuller MT, Nunnari J and Shaw JM (1998) Mitochondrial fusion in yeast requires the transmembrane GTPase Fzo1p. J. Cell. Biol. 143: 359-373

54. Santel A and Fuller M (2001) Control of mitochondrial morphology by a human mitofusin. J. Cell. Sci. 114: 867-874

55. Chen H, Detmer SA, Ewald AJ, Griffin EE, Fraser SE and Chan DC (2003) Mitofusins Mfn1 and Mfn2 coordinately regulate mitochondrial fusion and are essential for embryonic development. J. Cell. Biol. 160: 189-200

56. Rojo M, Legros F, Chateau D and Lombes A (2002) Membrane topology and mitochondrial targeting of mitofusins, ubiquitous mammalian homologs of the transmembrane GTPase Fzo. J. Cell. Sci. 115: 1663-1674

57. Fritz S, Rapaport D, Klanner E, Neupert W and Westermann B (2001) Connection of the mitochondrial outer and inner membranes by Fzo1 is critical for organellar fusion. J. Cell. Biol. 152: 683-692

58. Karbowski M, Lee Y-J, Gaume B, Jeong S-Y, Frank S, Nechushtan A, Santel A, Fuller M, Smith CL and Youle RJ. (2002) Spatial and temporal association of Bax with mitochondrial fission sites, Drp1, and Mfn2 during apoptosis. J. Cell. Biol. 159: 931-938

59. Sesaki H and Jensen RE (2001) UGO1 Encodes an Outer Membrane Protein Required for Mitochondrial Fusion. J. Cell. Biol. 152: 1123-1134

60. Olichon A, Emorine LJ, Descoins E, Pelloquin L, Brichese L, Gas N, Guillou E, Delettre C, Valette A, Hamel CP, Ducommun B, Lenaers $G$ and Belenguer $P$ (2002) The human dynamin-related protein OPA1 is anchored to the mitochondrial inner membrane facing the inter-membrane space. FEBS Lett. 523: $171-176$
61. Zamzami N, Marchetti P, Castedo M, Zanin C, Vayssiere JL, Petit PX and Kroemer G. (1995) Reduction in mitochondrial potential constitutes an early irreversible step of programmed lymphocyte death in vivo. J. Exp. Med. 181: 1661-1672

62. Letai A, Bassik MC, Walensky LD, Sorcinelli MD, Weiler S and Korsmeyer SJ (2002) Distinct BH3 domains either sensitize or activate mitochondrial apoptosis, serving as prototype cancer therapeutics. Cancer Cell. 2: 183-192

63. Wei MC, Zong W-X, Cheng EH-Y, Lindsten T, Panoutsakopoulou V, Ross AJ, Roth KA, MacGregor GR, Thompson CB and Korsmeyer SJ. (2001) Proapoptotic BAX and BAK: a requisite gateway to mitochondrial dysfunction and death. Science 292: 727-730

64. Shimizu S, Narita M and Tsujimoto Y (1999) Bcl-2 family proteins regulate the release of apoptogenic cytochrome $c$ by the mitochondrial channel VDAC. Nature 399: 483-487

65. Marzo I, Brenner C, Zamzami N, Jurgensmeier JM, Susin SA, Vieira HL, Prevost MC, Xie Z, Matsuyama S, Reed JC and Kroemer G (1998) Bax and adenine nucleotide translocator cooperate in the mitochondrial control of apoptosis. Science 281: 2027-2031

66. Antonsson B, Conti F, Ciavatta A, Montessuit S, Lewis S, Martinou I, Bernasconi L, Bernard A, Mermod JJ, Mazzei G, Maundrell K, Gambale F, Sadoul R and Martinou JC (1997) Inhibition of Bax channel-forming activity by Bcl-2. Science 277: 370-372

67. Schlesinger PH, Gross A, Yin XM, Yamamoto K, Saito M, Waksman G and Korsmeyer SJ (1997) Comparison of the ion channel characteristics of proapoptotic BAX and antiapoptotic BCL-2. Proc. Natl. Acad. Sci. USA 94: $11357-11362$

68. Kluck RM, Esposti MD, Perkins G, Renken C, Kuwana T, Bossy-Wetzel E, Goldberg M, Allen T, Barber MJ, Green DR and Newmeyer DD (1999) The proapoptotic Proteins, Bid and Bax, cause a limited permeabilization of the mitochondrial outer membrane that is enhanced by cytosol. J. Cell. Biol. 147: 809-822

69. von Ahsen O, Renken C, Perkins G, Kluck RM, Bossy-Wetzel E and Newmeyer DD (2000) Preservation of mitochondrial structure and function after Bid- or Bax-mediated cytochrome $c$ release. J. Cell. Biol. 150: 1027-1036

70. Scorrano L, Ashiya M, Buttle K, Weiller S, Oakes SA, Mannella CA and Korsmeyer SJ (2002) A distinct pathway remodels mitochondrial cristae and mobilizes cytochrome $c$ during apoptosis. Dev. Cell. 2: 55-67

71. Esposti MD, Erler JT, Hickman JA and Dive C (2001) Bid, a widely expressed proapoptotic protein of the Bcl-2 family, displays lipid transfer activity. Mol. Cell. Biol. 21: 7268-7276

72. Epand RF, Martinou J-C, Fornallaz-Mulhauser M, Hughes DW and Epand RM (2002) The apoptotic protein tBid promotes leakage by altering membrane curvature. J. Biol. Chem. 277: 32632-32639

73. Paumard P, Vaillier J, Coulary B, Schaeffer J, Soubannier V, Mueller DM, Brethes D, di Rago J-P and Velours J (2002) The ATP synthase is involved in generating mitochondrial cristae morphology. EMBO J. 21: 221-230

74. Olichon A, Baricault L, Gas N, Guillou E, Valette A, Belenguer P and Lenaers G (2002) Loss of OPA1 perturbates the mitochondrial inner membrane structure and integrity, leading to cytochrome $c$ release and apoptosis. J. Biol. Chem. 278: 7743-7746

75. Desagher S and Martinou JC (2000) Mitochondria as the central control point of apoptosis. Trends Cell. Biol. 10: 369-377

76. Capano $M$ and Crompton M (2002) Biphasic translocation of Bax to mitochondria. Biochem. J. 367: 169-178

77. Frank S, Gaume B, Bergmann-Leitner ES, Leitner WW, Robert EG, Catez F, Smith CL and Youle RJ (2001) The role of dynamin-related protein 1, a mediator of mitochondrial fission, in apoptosis. Dev. Cell. 1: 515-525

78. Pinton P, Ferrari D, Rapizzi E, Virgilio FD, Pozzan T and Rizzuto R (2001) The $\mathrm{Ca}^{2+}$ concentration of the endoplasmic reticulum is a key determinant of ceramide-induced apoptosis: significance for the molecular mechanism of Bcl-2 action. EMBO J. 20: 2690-2701

79. Rapizzi E, Pinton P, Szabadkai G, Wieckowski MR, Vandecasteele G, Baird G, Tuft RA, Fogarty KE and Rizzuto R. (2002) Recombinant expression of the voltage-dependent anion channel enhances the transfer of $\mathrm{Ca}^{2+}$ microdomains to mitochondria. J. Cell Biol. 159: 613-624

80. Mancini M, Anderson BO, Caldwell E, Sedghinasab M, Paty PB and Hockenbery DM. (1997) Mitochondrial proliferation and paradoxical membrane 
depolarization during terminal differentiation and apoptosis in a human colon carcinoma cell line. J. Cell. Biol. 138: 449-469

81. Chen W, Calvo PA, Malide D, Gibbs J, Schubert U, Bacik I, Basta S, O'Neill R Schickli J, Palese P, Henklein P, Bennink JR and Yewdell JW (2001) A novel influenza $A$ virus mitochondrial protein that induces cell death. Nat. Med. 7: 1306-1312

82. Breckenridge DG, Stojanovic M, Marcellus RC and Shore GC (2003) Caspase cleavage product of BAP31 induces mitochondrial fission through endoplasmic reticulum calcium signals, enhancing cytochrome $c$ release to the cytosol. J. Cell. Biol. 160: 1115-1127

83. Wolter KG, Hsu YT, Smith CL, Nechushtan A, Xi XG and Youle RJ (1997) Movement of Bax from the cytosol to mitochondria during apoptosis. J. Cell. Biol. 139: 1281-1292

84. Nechushtan A, Smith CL, Lamensdorf I, Yoon S-H and Youle RJ (2001) Bax and Bak coalesce into novel mitochondria-associated clusters during apoptosis. J. Cell. Biol. 153: 1265-1276

85. Cuddeback SM, Yamaguchi H, Komatsu K, Miyashita T, Yamada M, Wu C Singh S and Wang H-G (2001) Molecular cloning and characterization of Bif-1. A NOVEL Src Homology 3 Domain-CONTAInING PROtein that AsSOciates WITH Bax. J. Biol. Chem. 276: 20559-20565

86. Pierrat B, Simonen M, Cueto M, Mestan J, Ferrigno P and Heim J (2001) SH3GLB, a new endophilin-related protein family featuring an $\mathrm{SH} 3$ domain. Genomics 71: 222-234

87. Farsad K, Ringstad N, Takei K, Floyd SR, Rose K and De Camilli P. (2001) Generation of high curvature membranes mediated by direct endophilin bilayer interactions. J. Cell. Biol. 155: 193-200

88. Yamaguchi $\mathrm{H}$, Paranawithana SR, Lee MW, Huang Z, Bhalla KN and Wang $\mathrm{H}$ G (2002) Epothilone B analogue (BMS-247550)-mediated cytotoxicity through induction of bax conformational change in human breast cancer cells. Cancer Res. 62: 466-471

89. Ringstad N, Gad H, Low P, Di Paolo G, Brodin L, Shupliakov O and De Camilli P (1999) Endophilin/SH3p4 is required for the transition from early to late stages in clathrin-mediated synaptic vesicle endocytosis. Neuron 24: 143-154

90. Schmidt A, Wolde M, Thiele C, Fest W, Kratzin H, Podtelejnikov AV, Witke W, Huttner WB and Soling HD (1999) Endophilin I mediates synaptic vesicle formation by transfer of arachidonate to lysophosphatidic acid. Nature 401 : 133-14

91. Hsu YT and Youle RJ (1998) Bax in murine thymus is a soluble monomeric protein that displays differential detergent-induced conformations. J. Biol. Chem. 273: 10777-10783

92. Basanez G, Sharpe JC, Galanis J, Brandt TB, Hardwick JM and Zimmerberg J. (2002) Bax-type apoptotic proteins porate pure lipid bilayers through a mechanism sensitive to intrinsic monolayer curvature. J. Biol. Chem. 277: 49360-49365

93. Nemoto $Y$ and De Camilli $P$ (1999) Recruitment of an alternatively spliced form of synaptojanin 2 to mitochondria by the interaction with the PDZ domain of a mitochondrial outer membrane protein. EMBO J. 18: 2991-3006

94. Duncan CJ, Greenaway HC, Publicover SJ, Rudge MF and Smith JL (1980) Experimental production of 'septa' and apparent subdivision of muscle mitochondria. J. Bioenerg. Biomembr. 12: 13-33

95. Csordas G, Madesh M, Antonsson B and Hajnoczky G (2002) tcBid promotes $\mathrm{Ca}^{2+}$ signal propagation to the mitochondria: control of $\mathrm{Ca}^{2+}$ permeation through the outer mitochondrial membrane. EMBO J. 21 : 2198-2206

96. McCormick AL, Smith VL, Chow D and Mocarski ES. (2003) Disruption of mitochondrial networks by the human cytomegalovirus UL37 gene product viral mitochondrion-localized inhibitor of apoptosis. J. Virol. 77: 631-641

97. Goldmacher VS, Bartle LM, Skaletskaya A, Dionne CA, Kedersha NL, Vater CA, Han J-w, Lutz RJ, Watanabe S, Cahir McFarland ED, Kieff ED, Mocarsk ES and Chittenden T. (1999) A cytomegalovirus-encoded mitochondrialocalized inhibitor of apoptosis structurally unrelated to Bcl-2. Proc. Natl. Acad. Sci USA 96: 12536-12541

98. Colberg-Poley AM, Patel MB, Erezo DP and Slater JE (2000) Human cytomegalovirus UL37 immediate-early regulatory proteins traffic through the secretory apparatus and to mitochondria. J. Gen. Virol. 81: 1779-1789

99. Kowaltowski AJ, Cosso RG, Campos CB and Fiskum G (2002) Effect of Bcl-2 overexpression on mitochondrial structure and function. J. Biol. Chem. 277: 42802-42807 